Original paper

\title{
Monte Carlo-based dose calculation engine for minibeam radiation therapy
}

\author{
I. Martínez-Rovira ${ }^{\text {a,b,c,* }}$, J. Sempau ${ }^{\text {b,d }}$, Y. Prezado $^{\mathrm{e}}$ \\ a Service Hospitalier Frédéric Joliot (DSV/I2BM/SHFJ), Commissariat à l'Énergie Atomique et aux énergies alternatives (CEA), 4, Place du Général Leclerc, \\ F-91401 Orsay, France \\ ${ }^{\mathrm{b}}$ Institut de Tècniques Energètiques (INTE), Universitat Politècnica de Catalunya (UPC), Diagonal 647, E-08028 Barcelona, Spain \\ ${ }^{\mathrm{c}}$ ID17 Biomedical Beamline, European Synchrotron Radiation Facility (ESRF), B.P. 220, 6 rue Jules Horowitz, F-38043 Grenoble Cedex, France \\ ${ }^{\mathrm{d}}$ Networking Research Centre, CIBER-BBN, Barcelona, Spain \\ e Laboratoire Imagerie et Modélisation en Neurobiologie et Cancérologie, Centre National de la Recherche Scientifique (CNRS), 15 rue Georges Clemenceau, \\ Bât. 440F-91406 Orsay Cedex, France
}

\section{A R T I C L E I N F O}

\section{Article history:}

Received 14 September 2012

Received in revised form

25 February 2013

Accepted 28 February 2013

Available online 15 April 2013

\section{Keywords:}

Minibeam radiation therapy

Treatment planning system

Monte Carlo calculations

Spatially fractionated synchrotron

radiotherapy techniques

\begin{abstract}
A B S T R A C T
Minibeam radiation therapy (MBRT) is an innovative radiotherapy approach based on the wellestablished tissue sparing effect of arrays of quasi-parallel micrometre-sized beams. In order to guide the preclinical trials in progress at the European Synchrotron Radiation Facility (ESRF), a Monte Carlobased dose calculation engine has been developed and successfully benchmarked with experimental data in anthropomorphic phantoms. Additionally, a realistic example of treatment plan is presented. Despite the micron scale of the voxels used to tally dose distributions in MBRT, the combination of several efficiency optimisation methods allowed to achieve acceptable computation times for clinical settings (approximately $2 \mathrm{~h}$ ). The calculation engine can be easily adapted with little or no programming effort to other synchrotron sources or for dose calculations in presence of contrast agents.
\end{abstract}

(C) 2013 Associazione Italiana di Fisica Medica. Published by Elsevier Ltd. All rights reserved.

\section{Introduction}

Minibeam radiation therapy (MBRT) is a novel radiotherapy (RT) technique based on a distinct dose delivery method which uses submillimetric field sizes $(500-700 \mu \mathrm{m})$ in combination with the spatial fractionation of the dose. In MBRT, the irradiation is performed with arrays of kilovoltage X-rays beams spaced by around $1.2 \mathrm{~mm}$ [1]. The concept was first proposed by Dilmanian et al. (2006) and, since 2008, it has been explored at the ID17 Biomedical Beamline of the European Synchrotron Radiation Facility (ESRF). The resulting biological effects appear to challenge many of the current paradigms in RT. In particular, a remarkable healthy tissue resistance to very high doses in one fraction was observed in several preclinical investigations [2,3]. Additionally, this type of irradiations rendered significant tumour growth delay $[3,4]$.

\footnotetext{
* Corresponding author. Service Hospitalier Frédéric Joliot (DSV/I2BM/SHFJ), Commissariat à l'Énergie Atomique et aux énergies alternatives (CEA), 4, Place du Général Leclerc, F-91401 Orsay, France.

E-mail address: immamartinez@gmail.com (I. Martínez-Rovira).
}

In contrast with other synchrotron RT techniques like microbeam radiation therapy (MRT) (see Refs. [5-7], among others), MBRT has potential for widespread clinical implementation. Wider beams are used to eliminate the need for extremely short irradiation times (and therefore, high dose rates) in order to prevent beam smearing, as it is the case of MRT. Hence, it is conceptually possible to extend this technique by using modified X-ray equipment, creating an opportunity for its implementation at hospitals [2]. In addition, it is feasible to use higher beam energies $(\geq 200 \mathrm{keV})$, which would lead to lower entrance doses for a given dose to the tumour [8]. Moreover, the interlacing of two orthogonal arrays of minibeams that produce a homogeneous dose in the tumour is easier in MBRT [9], while the mechanical precision required in MRT makes such kind of irradiations extremely difficult.

The aforementioned advantages triggered the exploration of MBRT at the ESRF [3,4], where MBRT preclinical trials have started in order to evaluate the tumour and healthy tissue responses. If successful, these preclinical trials would open the way to the future clinical trials in MBRT, similarly to what happened in MRT [10]. Due to the complexity of these techniques, a preliminary step is planned in MRT when moving from the preclinical trials with rodents to the 
clinical trials in humans, which consists in the irradiation of large animals (cats and dogs) [11].

Within this framework, an accurate dose computation software is required to guide the forthcoming MBRT experiments. The peculiar irradiation characteristics, as compared to conventional RT (synchrotron source, beam modifiers, energy spectrum, micrometre-sized fields, etc.), demanded the development of a dedicated dose calculation tool [12,13]. Thus, the aim of this study was the development and experimental benchmarking of a Monte Carlo (MC)-based calculation engine devoted to MBRT. This computation engine includes the complete characterisation and modelling of the synchrotron source and the evaluation of dose distributions in a realistic voxelised model of the patient for one or several (interlaced) arrays.

It is important to note that, despite a previous MBRT dosimetric study has been published including dose calculations in complex structures [14], it does not include neither an accurate source model nor an experimental verification. The dose calculation engine developed in this work, together with the previous developed dosimetry protocol [9] constitute the full set of dosimetry tools required to carry out preclinical and, eventually, clinical trials in MBRT in a reliable and reproducible way. Finally, an estimation of the calculation times in a realistic treatment scenario envisaged in (possible) future clinical trials in MBRT has been evaluated for the first time.

\section{Materials and methods}

\section{Radiation source and minibeams generation}

The ESRF is a third-generation synchrotron with a $6.04 \mathrm{GeV}$ electron storage ring. Electrons are deflected in devices consisting of a series of magnets, which produce extremely bright X-ray beams. In the particular case of ID17, the device is a wiggler. The generated synchrotron radiation traverses different types of beam modifiers until it reaches the patient position (see Fig. 1), where targets are placed on a high-precision goniometer. The beam characteristics change along its path; the mean energy at the patient position is approximately $100 \mathrm{keV}$, while the maximum beam dimensions are $2.5 \mathrm{~mm}$ in height and $41 \mathrm{~mm}$ in width [12]. In order to fill the desired vertical irradiation field size, the target is vertically scanned through the beam [15].

The vertical spatial fractionation of the beam is achieved by using a special device called white-beam chopper, which is composed by ten $600-\mu \mathrm{m}$-wide tungsten carbide blades [16]. This innovative method offers an excellent reproducibility [1].

\section{Source modelling}

The ID17 X-ray source (wiggler) was modelled by the synchrotron ray tracing code SHADOW [17] and the MC simulation code PENELOPE [18] was employed to transport the photon beam from the source to the patient position through the beamline. The latter code allows the simulation of the coupled transport of electrons, photons, and positrons in a wide energy range and in arbitrary material systems. The two codes were directly linked by inserting a new source model into the general-purpose main program for PENELOPE named penEasy [19].

The phase-space state variables (energy, position, direction of flight and polarisation state) of the particles were saved in a phasespace file (PSF) at the position marked in Fig. 1. The information contained in this PSF was used as an input to generate an analytical photon beam source model. First, the photon energy was sampled from the MC calculated spectrum, which ranged from 27 to $600 \mathrm{keV}$ with a mean energy of $99 \mathrm{keV}$. Secondly, a completely linearly polarised beam (along the electron orbital plane) was considered. Finally, the probability distribution of the horizontal coordinate was approximated as a uniform distribution with a width equal to the field size, while the joint distribution for position $(x)$ and divergence $(u)$ was approximated by a straight line obtained by the linear regression $u=a x$, where $a=0.000256(4) \mathrm{cm}^{-1}$ is the regression coefficient.

Particles were then transported through the chopper and the vertical slit (see Fig. 1); the vertical scanning of the beam has also been considered. Further details can be found in our previous work [12].

\section{MC simulation tool for dose computations in voxelised phantoms}

Dose distributions in several phantoms were computed for a photon beam sampled according to the probability distributions derived from the beam model. The MC code PENELOPE/penEasy was used for dose computation.

Several modifications were performed on the code in order to be able to assess dose distributions at micron scale in a voxelised model of a patient, which is typically obtained from computed tomography (CT) images. For instance, the decoupling of the CT image voxel grid (lateral voxel size in the order of $\mathrm{mm}$ ) to the dose

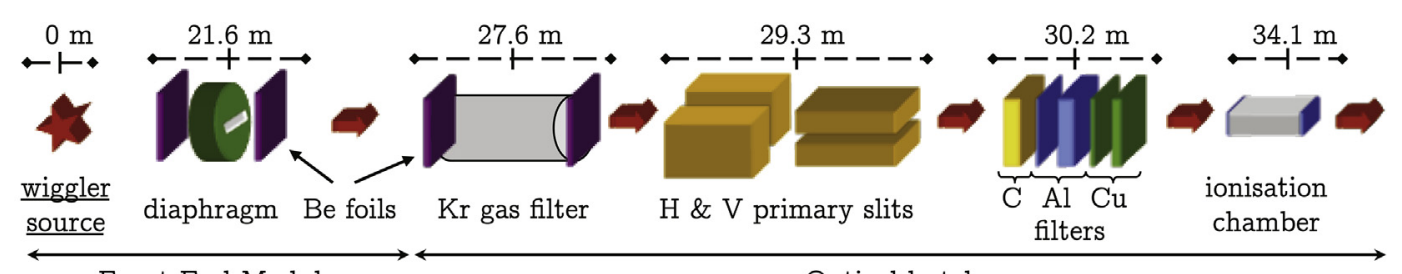

Front End Module

Optical hutch

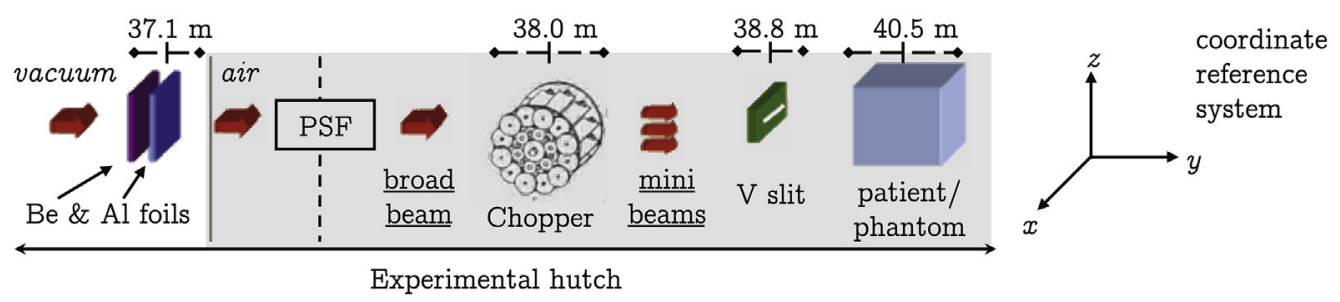

Figure 1. Scheme of the ID17 beamline elements from the wiggler to the patient position (not to scale). Adapted from Refs. [12,16]. 

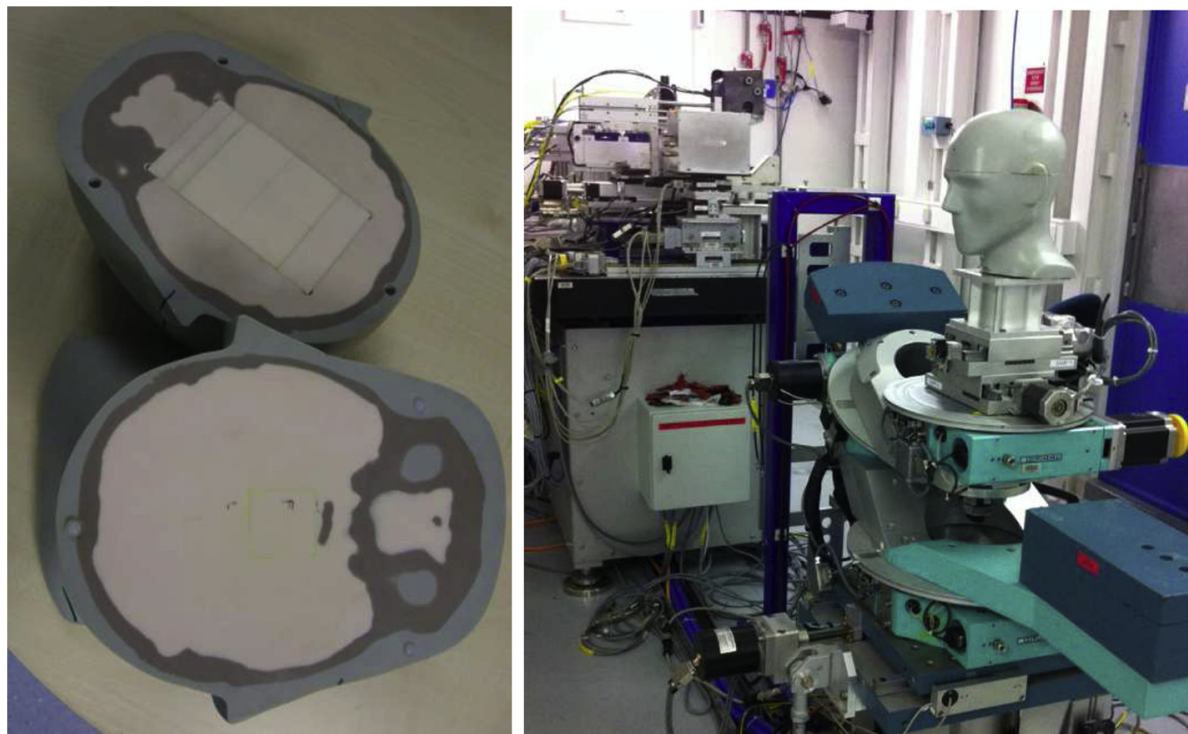

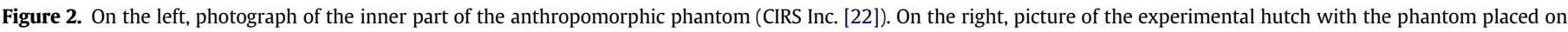
the high-precision goniometer.

bin grid (required lateral bin size in the order of $100 \mu \mathrm{m}$ in the transversal direction of the minibeams) was performed. Additionally, a program was developed to orientate the CT image along the direction of the minibeams for a given irradiation angle [13].

Typically, the attenuation map represented in the CT images is given in Hounsfield Units (HU). However, most MC codes require knowing the mass density and material index of each voxel. To convert HU to mass density, the calibration curve provided by the hospital where the CT was taken is used. The material index of each voxel was obtained by using a classification approach, that is, defining a mapping between $\mathrm{HU}$ intervals and the material index [20].

Several optimisation methods were applied in order to speed up the simulations. In particular, interaction forcing was applied to photon transport [18]. Additionally, simulations were parallelised by using independent random sequences $[13,21]$.

\section{MC calculations and experimental validation in an} anthropomorphic phantom

MC calculations were experimentally verified by using radiochromic films (Gafchromic HD-810) analysed with a flat bed scanner (Epson Perfection V750-M Pro Scanner). Dose calculations had already been successfully validated in homogeneous and heterogeneous slab phantoms in the work of Prezado et al. [9]. In this

\section{Table 1}

Calculated (MC) versus measured (Film) central PVDR at different depths in an anthropomorphic phantom. MBRT parameters: unidirectional; $570-\mu \mathrm{m}$-wide minibeams spaced by $1.2 \mathrm{~mm}$; field sizes: $1 \times 1,2 \times 2$ and $3 \times 3 \mathrm{~cm}^{2}$. '(lat)' and '(AP)' denote a lateral and anterio-posterior irradiation, respectively. Statistical uncertainties correspond to $2 \sigma$.

\begin{tabular}{|c|c|c|c|c|c|c|}
\hline \multicolumn{7}{|l|}{ PVDR } \\
\hline \multirow[t]{2}{*}{ Depth $(\mathrm{cm})$} & \multicolumn{2}{|l|}{$1 \times 1 \mathrm{~cm}^{2}$} & \multicolumn{2}{|l|}{$2 \times 2 \mathrm{~cm}^{2}$} & \multicolumn{2}{|c|}{$3 \times 3 \mathrm{~cm}^{2}$} \\
\hline & $\mathrm{MC}$ & Film & MC & Film & $\mathrm{MC}$ & Film \\
\hline 4.4 (lat) & $12.8 \pm 0.2$ & $13 \pm 1$ & $6.6 \pm 0.1$ & $6.5 \pm 0.7$ & $5.2 \pm 0.1$ & $5.3 \pm 0.5$ \\
\hline 10.8 (lat) & $11.9 \pm 0.3$ & $11 \pm 1$ & $6.3 \pm 0.2$ & $6.5 \pm 0.7$ & $4.5 \pm 0.1$ & $4.6 \pm 0.5$ \\
\hline $5.6(\mathrm{AP})$ & $12.9 \pm 0.2$ & $12 \pm 1$ & $6.6 \pm 0.1$ & $6.4 \pm 0.6$ & $4.8 \pm 0.1$ & $4.2 \pm 0.4$ \\
\hline $7.6(\mathrm{AP})$ & $12.5 \pm 0.3$ & $12 \pm 1$ & $6.4 \pm 0.2$ & $6.2 \pm 0.6$ & $4.4 \pm 0.1$ & $5.0 \pm 0.5$ \\
\hline
\end{tabular}

study, simulations and measurements were performed in a realistic anthropomorphic head phantom [22], which mimics the tissues with approximately $1 \%$ accuracy in our energy range (data provided by CIRS Inc. [22]). See Fig. 2. This phantom has already been used in previous dosimetry works [23].

CT images of this phantom were used as an input for the MC calculations. Dose distributions were tallied in voxels of $100 \mu \mathrm{m}$ along the transversal direction of the minibeams, while the bin size was set to the voxel size in the other two dimensions (CT voxel size: $0.35 \times 1.00 \times 0.35 \mathrm{~mm}^{3}$ ). From these distributions, the ratio between the peak dose (dose at the center of one minibeam) and the valley dose (dose in the central flat region of $300 \mu \mathrm{m}$ between minibeams), also named peak-to-valley dose ratio (PVDR), was assessed. This parameter is one of the most relevant quantities in this type of spatially fractionated synchrotron RT techniques [5].

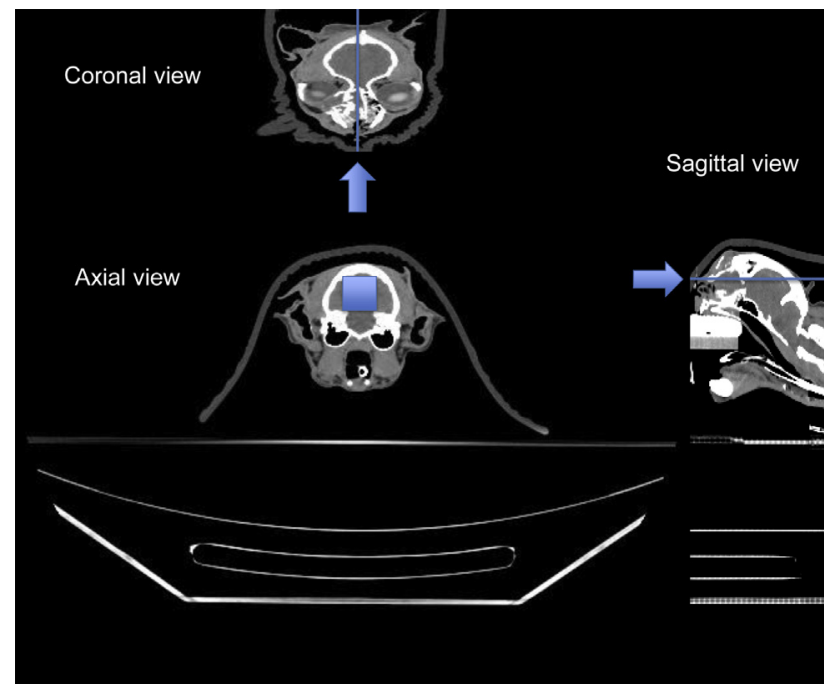

Figure 3. Coronal, axial and sagittal view of the irradiated dog's head. The arrow represents the beam direction (AP) and the line indicates the central part of the beam in depth. The square in the axial view corresponds to the whole irradiation field size $\left(2 \times 2 \mathrm{~cm}^{2}\right)$ 


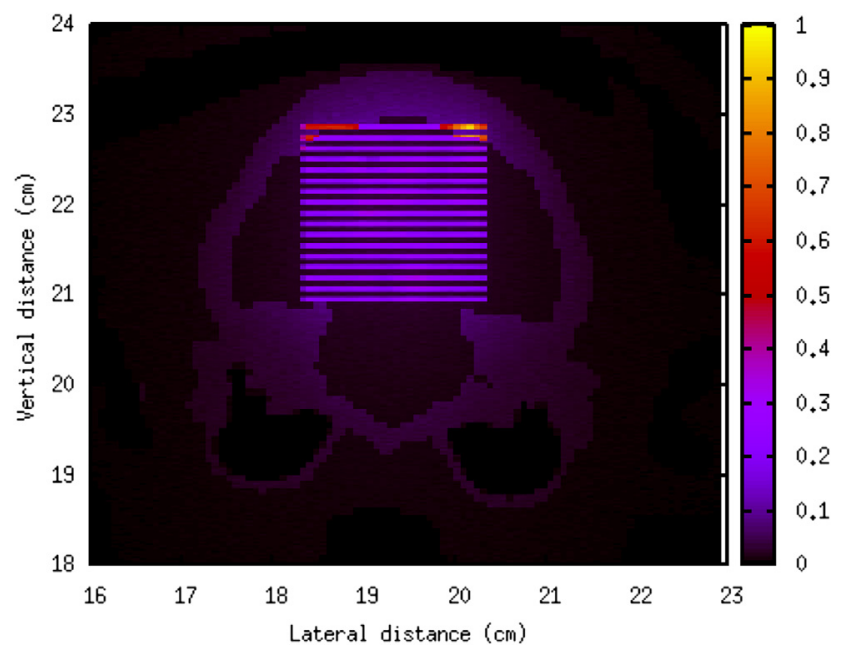

Figure 4. Dose map (arbitrary units, a.u.) at a depth of $9 \mathrm{~cm}$ from the dog's head surface. MBRT parameters: AP unidirectional; $570-\mu \mathrm{m}$-wide minibeams spaced by $1.2 \mathrm{~mm}$; field size: $2 \times 2 \mathrm{~cm}^{2}$.

PVDR values were evaluated at several depths for different field sizes $\left(1 \times 1,2 \times 2\right.$ and $\left.3 \times 3 \mathrm{~cm}^{2}\right)$. These field sizes are consistent with previous synchrotron RT dosimetry works [9,12,24,25]. For lateral irradiations, films were placed at the depths of 4.4 and $10.8 \mathrm{~cm}$ from the phantom surface and for anterior-posterior (AP) irradiations, at the depths of 5.5 and $7.6 \mathrm{~cm}$. Results are presented in Section Experimental validation of the MC calculations in an anthropomorphic phantom.

\section{MC simulations of a realistic treatment plan}

An example of a dose calculation in a dog's head will be presented in Section MC calculations in voxelised structures: an example of a realistic treatment plan. Voxels of CT image of the dog were classified into three materials: soft tissue, skull, and air. Soft tissue was identified as water with a varying mass density. The skull composition was extracted from the International Commission on Radiation Units and Measurements (ICRU) Report 46 [26].

Two irradiation modalities were evaluated: unidirectional (AP and lateral) and interlaced arrays. In both cases, the MBRT irradiation parameters were fixed to $570-\mu \mathrm{m}$-wide minibeams spaced by $1.2 \mathrm{~mm}$, which showed a perfect interlaced pattern in a homogeneous phantom [9]. The whole irradiation field size was set to $2 \times 2 \mathrm{~cm}^{2}$. Dose was tallied in bins of

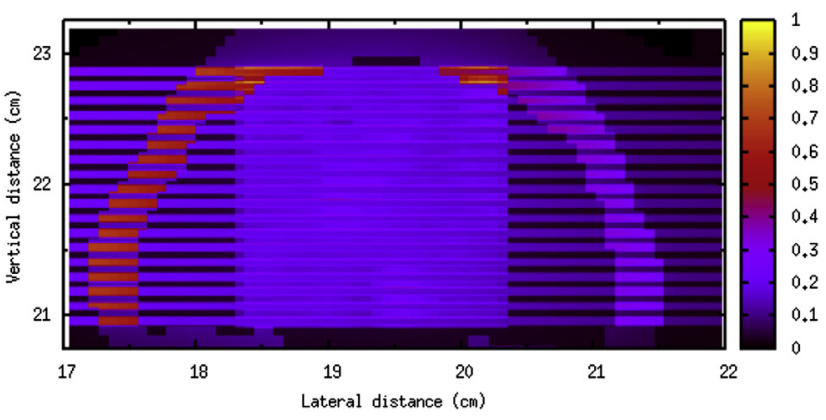

Figure 6. Dose map (a.u.) at a depth of $9 \mathrm{~cm}$ from the dog's head surface for an interlaced irradiation geometry. MBRT parameters: AP and lateral irradiations; $570-\mu \mathrm{m}-$ wide minibeams spaced by $1.2 \mathrm{~mm}$; field size: $2 \times 2 \mathrm{~cm}^{2}$.

$0.74 \mathrm{~mm}$ (lateral direction of the minibeams) $\times 100 \mu \mathrm{m}$ (transversal direction of the minibeams) $\times 1.25 \mathrm{~mm}$ (depth) (CT voxel size: $0.74 \times 0.74 \times 1.25 \mathrm{~mm}^{3}$ ).

\section{Results and discussion}

Experimental validation of the MC calculations in an anthropomorphic phantom

Table 1 shows the calculated and measured central PVDR values as a function of depth in an anthropomorphic phantom for several field sizes (see details in Section MC calculations and experimental validation in an anthropomorphic phantom). A decrease of PVDR is observed when the field size is increased. Additionally, PVDR values are similar to those found in a homogeneous phantom [15] due to the fact that the radiochromic film positions (limited by the phantom geometry) are far from the bone heterogeneity.

MC calculations reproduce experimental results within uncertainty bars even in such complex phantoms, showing the performance of the developed MC calculation tool for its use in MBRT dose calculations. It is important to note that, for the first time, dose distributions in MBRT were validated in an anthropomorphic phantom.

\section{MC calculations in voxelised structures: an example of a realistic treatment plan}

Dose calculations were performed in a voxelised model of a dog's head obtained from a CT scan (see Fig. 3). The minibeam dose pattern is clearly visible in Fig. 4, which shows an axial dose map for an AP unidirectional irradiation (field size of $2 \times 2 \mathrm{~cm}^{2}$ ).
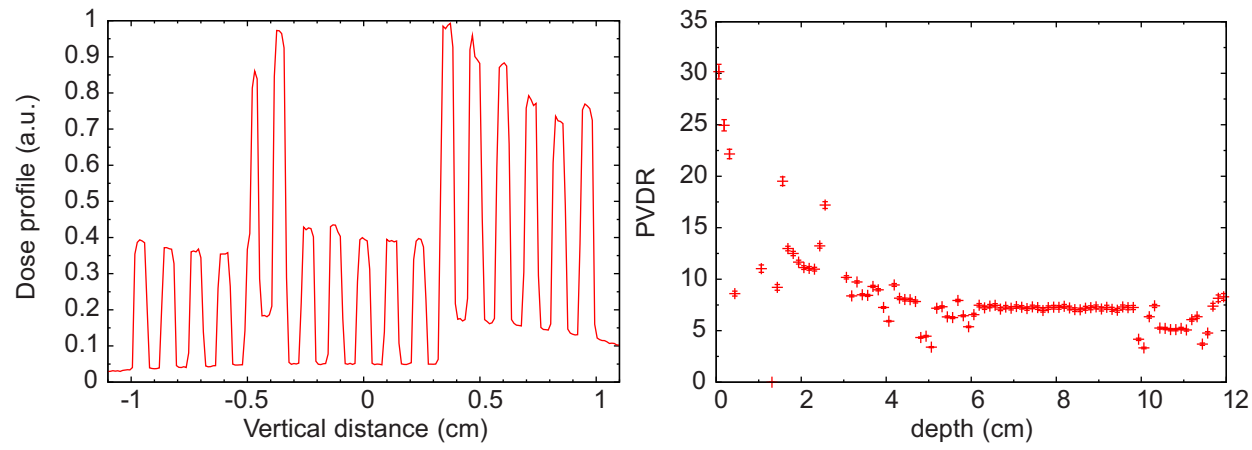

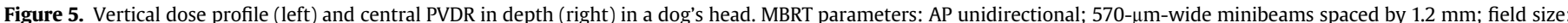

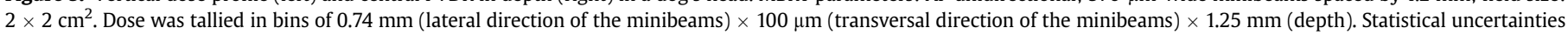
correspond to $2 \sigma$. 


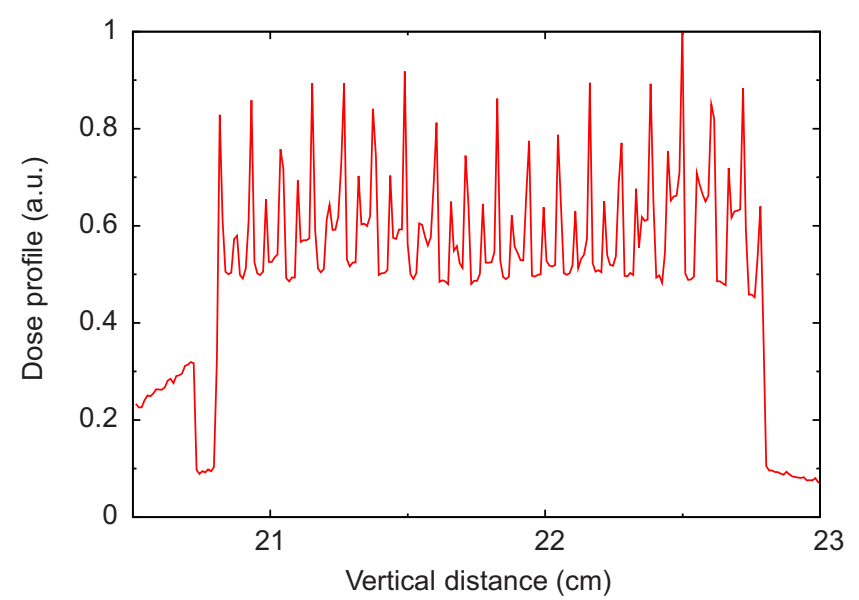

Figure 7. Vertical dose profile at a depth of $9 \mathrm{~cm}$ from the dog's head surface for an interlaced irradiation geometry. MBRT parameters: AP and lateral irradiations; 570$\mu \mathrm{m}$-wide minibeams spaced by $1.2 \mathrm{~mm}$; field size: $2 \times 2 \mathrm{~cm}^{2}$.

In this type of spatially fractionated RT techniques, quantitative information on peak and valley doses and the corresponding PVDR is needed. The left part of Fig. 5 presents a lateral dose profile (at a depth of $4.5 \mathrm{~cm}$ ), whereas the right part shows the PVDR values in depth. The enhancement in both the peak and the valley doses (and the corresponding reduction in the PVDR values) is due to the presence of the bone. PVDR values are not uniform in the target volume due to the important tissue heterogeneities encountered by the minibeams in their path.

The calculation time to reach a $2 \%$ uncertainty $(2 \sigma)$ in the central valley dose at a depth of $4.5 \mathrm{~cm}$ (from the dog's head surface) on a $2.93 \mathrm{GHz}$ Intel Xeon Processor X5670 (Compiler: Intel Fortran v. 10.0 , option-03) is 3.4 days. By parallelising the simulations in 40 cores, the computation time is reduced to $2 \mathrm{~h}$. These values comply with the accuracy of $2-5 \%$ in dose delivery that is required to effectively treat certain types of cancers and to reduce complications, as reflected in ICRU Reports 24 and 42 [27,28]. Additionally, these calculation times are perfectly acceptable in such clinical conditions (it is important to note that the flexibility in the timings for clinical trials in synchrotrons is higher than in a clinical environment).

The computation engine also enables the assessment of dose distributions in bidirectional irradiations (or other irradiation modalities). In fact, as it was shown in preclinical trials [2-4], interlacing of two beams is needed in order to increase the valley doses in the target and, thus, achieve a high enough density of lesions in the tumour, while healthy tissues still benefit from the spatial fractionation of the dose. Figure 6 shows the dose map for a bidirectional irradiation interlaced at a depth of $9 \mathrm{~cm}$ from the dog's head surface.

Figure 7 shows the vertical dose profile at the centre of the target. One can see that the irradiation parameters that lead to a homogeneous interlaced dose distributions [9] produce hot regions in the target when irradiating realistic geometries. Previous studies revealed that the overlapping parts producing hot spots in the tumour (some tens of microns) should not produce deleterious effects $[3,29,30]$.

\section{Conclusion}

MBRT is an innovative spatially fractionated synchrotron RT technique with potential applications using sources other than synchrotrons. To guide the biological studies, an MC-based computation engine has been developed and successfully benchmarked with experimental data in complex phantoms. An example of treatment planning scenario has been presented, showing the performance of the calculation engine. The final computation time (approximately $2 \mathrm{~h}$ ) is acceptable for clinical trials in synchrotrons. This calculation engine can be easily adapted with little or no programming effort to other synchrotrons or for dose calculations in presence of contrast agents [31]. The developments performed in this work, along with the previously developed dosimetry protocol [9], constitute the full set of dosimetry tools required to carry out preclinical studies and, eventually, clinical trials in MBRT in a reliable and reproducible way.

\section{Acknowledgements}

The authors warmly thank the ESRF and the ID17 team, for support and granted beamtime. In particular, we are very grateful to H Requardt. Thanks to G Foerstner from the Systems and Communications group (ESRF) for her help in the use of the ESRF computing cluster. Many thanks to M Vautrin, P Deman and JF Adam (INSERM U836) and to A Sumova and B Kaser-Hotz (AOI Center) for providing us with CT images of the anthropomorphic phantom and of the pets, respectively. JS acknowledges partial financial support from the Spanish Ministerio de Economía y Competitividad (project no. FIS2012-38480).

\section{References}

[1] Prezado Y, Renier M, Bravin A. A new method of creating minibeam patterns for synchrotron radiation therapy: a feasibility study. J Synchrotron Radiat 2009;16:582-6.

[2] Dilmanian FA, Zhong Z, Bacarian T, Beneviste H, Romanelli P, Wang R, et al. Interlaced X-ray microplanar beams: a radiosurgery approach with clinical potential. Proc Natl Acad Sci U S A 2006;103:9709-14.

[3] Deman P, Vautrin M, Edouard M, Stupar V, Bobyk L, Farion R, et al. Monochromatic minibeams radiotherapy: from healthy tissue-sparing effect studies toward first experimental glioma bearing rats therapy. Int J Radiat Oncol Biol Phys 2012;82:693-700.

[4] Prezado Y, Sarun S, Gil S, Deman P, Bouchet A, Le Duc G. Increase of lifespan for glioma-bearing rats by using minibeam radiation therapy. J Synchrotron Radiat 2012;19:60-5.

[5] Dilmanian FA, Button TM, Le Duc G, Zhong N, Peña LA, Smith JA, et al. Response of rat intracranial 9L gliosarcoma to microbeam radiation therapy. Neuro Oncol 2002;4:26-38.

[6] Anschel DJ, Romanelli P, Benveniste H, Foerster B, Kalef-Ezra J, Zhong Z, et al. Evolution of a focal brain lesion produced by interlaced microplanar X-rays. Minim Invasive Neurosurg 2007;50:43-6.

[7] Bouchet A, Lemasson B, Le Duc G, Maisin C, Bräuer-Krisch E, Siegbahn EA, et al. Preferential effect of synchrotron microbeam radiation therapy on intracerebral 9L gliosarcoma vascular networks. Int J Radiat Oncol Biol Phys 2010;78: 1503-12.

[8] Prezado Y, Thengumpallil S, Renier M, Bravin A. X-ray energy optimization in minibeam radiation therapy. Med Phys 2009;36:4897-902.

[9] Prezado Y, Martínez-Rovira I, Thengumpallil S, Deman P. Dosimetry protocol for the preclinical trials in white-beam minibeam radiation therapy. Med Phys 2011;38:5012-20.

[10] Martínez-Rovira I, Sempau J, Fernández-Varea JM, Bravin A, Prezado Y. Monte Carlo dosimetry for forthcoming clinical trials in X-ray microbeam radiation therapy. Phys Med Biol 2010;55:4375-88.

[11] Braüer-Krisch E, Serduc R, Siegbahn EA, Le Duc G, Prezado Y, Bravin A, et al. Effects of pulsed, spatially fractionated, microscopic synchrotron X-ray beams on normal and tumoral brain tissue. Mutat Res 2010;704:160-6.

[12] Martínez-Rovira I, Sempau J, Prezado Y. Development and commissioning of a Monte Carlo photon beam model for the forthcoming clinical trials in microbeam radiation therapy. Med Phys 2012;39:119-31.

[13] Martínez-Rovira I, Sempau J, Prezado Y. Monte Carlo-based treatment planning system calculation engine for microbeam radiation therapy. Med Phys 2012;39:2829-38.

[14] Gokeri G, Kocar C, Tombakoglu M. Monte Carlo simulation of microbeam radiation therapy with an interlaced irradiation geometry and Au contrast agent in a realistic head phantom. Phys Med Biol 2010;55:7469-87.

[15] Prezado Y, Vautrin V, Martínez-Rovira I, Bravin A, Berkvens P, Adam JF. Dosimetry protocol for the forthcoming clinical trials in synchrotron radiation therapy (SSRT). Med Phys 2011;38:1709-17. 
[16] Renier M, Fiedler S, Nemoz C, Gonzalez H, Berruyer G, Bravin A. A mechanical chopper with continuously adjustable duty cycle for a wide X-ray beam. Nucl Instrum Methods A 2004;548:111-5.

[17] Sánchez del Río M, Canestrari N, Jiang F, Cerrina F. SHADOW3: a new version of the synchrotron X-ray optics modelling package. J Synchrotron Radiat 2011;18:1-9.

[18] Salvat F, Fernández-Varea JM, Sempau J. PENELOPE-2008, a code system for Monte Carlo simulation of electron and photon transport. Issy-les-Moulineaux: OECD Nuclear Energy Agency; 2008.

[19] Sempau J, Badal A, Brualla L. A PENELOPE-based system for the automated Monte Carlo simulation of clinacs and voxelized geometries - application to far-from-axis fields. Med Phys 2011;38:5887-95.

[20] Reynaert N, van der Marck SC, Schaart DR, van der Zee W, van VlietVroegindeweij C, Tomsej M, et al. Monte Carlo treatment planning for photon and electron beams. Radiat Phys Chem 2007;76:643-86.

[21] Badal A, Sempau J. A package of Linux scripts for the parallelization of Monte Carlo simulations. Comput Phys Commun 2006;175:440-50.

[22] CIRS Inc., Computed Imaging Reference Systems Inc. http://www.cirsinc.com/.

[23] Boudou C, Troprès I, Rousseau J, Lamalle L, Adam JF, Estève F, et al. Polymer gel dosimetry for synchrotron stereotactic radiotherapy and iodine doseenhancement measurements. Phys Med Biol 2007;52:4881-92.
[24] Stepanek J, Blattmann H, Laissue JA, Lyubimova N, Di Michiel M, Slatkin DN Physics study of microbeam radiation therapy with PSI-version of Monte Carlo code GEANT as a new computational tool. Med Phys 2000;27:1664-75.

[25] Siegbahn EA, Stepanek J, Bruer-Krisch E, Bravin A. Determination of dosimetrical quantities used in microbeam radiation therapy (MRT) with Monte Carlo simulations. Med Phys 2006;33:3248-59.

[26] ICRU 46. Photon, electron, proton and neutron interaction data for body tissues. Bethesda: ICRU; 1992.

[27] ICRU 24. Determination of absorbed dose in a patient irradiated by beams of $X$ or gamma rays in radiotherapy procedures. Bethesda: ICRU; 1976.

[28] ICRU 42. Use of computers in external beam radiotherapy procedures with high energy photons and electrons. Bethesda: ICRU; 1987.

[29] Serduc R, van de Looij YV, Francony G, Verdonck O, van der Sanden B Laissue J, et al. Characterization and quantification of cerebral edema induced by synchrotron X-ray microbeam radiation therapy. Phys Med Biol 2008;53: $1153-66$.

[30] Serduc R, Bräuer-Krisch E, Siegbahn EA, Bouchet A, Pouyatos B, Carron R, et al. High-precision radiosurgical dose delivery by interlaced microbeam arrays of high-flux low-energy synchrotron X-rays. Plos One 2010;5. e9028-1-12.

[31] Martínez-Rovira I, Prezado Y. Monte Carlo dose enhancement studies in microbeam radiation therapy. Med Phys 2011;38:4430-8. 